\title{
Multiple point statistical simulation using uncertain (soft) conditional data
}

Hansen, Thomas Mejer; Vu, Le Thanh; Mosegaard, Klaus; Cordua, Knud Skou

Published in:

Computers \& Geosciences

DOI:

10.1016/j.cageo.2018.01.017

Publication date:

2018

Document version

Peer reviewed version

Document license:

CC BY-NC-ND

Citation for published version (APA):

Hansen, T. M., Vu, L. T., Mosegaard, K., \& Cordua, K. S. (2018). Multiple point statistical simulation using uncertain (soft) conditional data. Computers \& Geosciences, 114, 1-10.

https://doi.org/10.1016/j.cageo.2018.01.017 
2

3

4

5

6

7 8

9 Abstract

Geostatistical simulation methods have been used to quantify spatial variability of reservoir models since the 80s. In the last two decades, state of the art simulation methods have changed from being based on covariance-based 2-point statistics to multiple-point statistics (MPS), that allow simulation of more realistic Earth-structures. In addition, increasing amounts of geoinformation (geophysical, geological, etc.) from multiple sources are being collected. This pose the problem of integration of these different sources of information, such that decisions related to reservoir models can be taken on an as informed base as possible. In principle, though difficult in practice, this can be achieved using computationally expensive Monte Carlo methods. Here we investigate the use of sequential simulation based MPS simulation methods conditional to uncertain (soft) data, as a computational efficient alternative. First, it is demonstrated that current implementations of sequential simulation based on MPS (e.g. SNESIM, ENESIM and Direct Sampling) do not account properly for uncertain conditional information, due to a combination of using only co-located information, and a random simulation path. Then, we suggest two approaches that better account for the available uncertain information. The first make use of a preferential simulation path, where more informed model parameters are visited preferentially to less informed ones. The second approach involves using non co-located uncertain information. For different types of available data, these approaches are demonstrated to produce simulation results similar to those obtained by the general Monte Carlo based approach. These methods allow MPS simu- 
lation to condition properly to uncertain (soft) data, and hence provides a computationally attractive approach for integration of information about a reservoir model.

Keywords: Multiple point statstics, Uncertain data, Data integration

\section{Introduction}

During the last 30 years a number of probabilistic based methods and algorithms have been developed in the geostatistical community, that allow quantification and simulation of increasingly geologically complex structural variability, see e.g. Deutsch and Journel (1992); Guardiano and Srivastava (1993); Strebelle (2000); Remy et al. (2008); Mariethoz et al. (2010); Straubhaar et al. (2011); Mariethoz and Kelly (2011); Toftaker and Tjelmeland (2013); Tahmasebi et al. (2014); Mariethoz and Caers (2014).

State of the art simulation methods have changed from being based on 2-point statistics (covariance-based statistics) to multiple-point statistics (MPS), that allow simulation of more realistic Earth-structures. MPS is especially important used as a base for flow modeling, as traditional 2-point statistics cannot adequately describe for example realistic connectivity of geological structures, that may have significant effect on flow properties and transport, see e.g. Zinn and Harvey (2003); Renard et al. (2011). The information about the expected spatial variability of the properties in a reservoir model can be conveniently provided in form of a 'training image'/'sample model' when using MPS. Using such a training image, several methods exist for simulation of multiple realizations of reservoir models that are consistent with the spatial statistics of the training image, e.g. Guardiano and Srivastava (1993); Strebelle (2000); Mariethoz et al. (2010).

Additional information is often available from e.g. boreholes and geophysical surveys (seismic, electromagnetic,..). Ideally, this information should be combined with the geostatistical information in order to obtain a stochastic reservoir model, or realizations of such a model that are consistent with all available data/information.

Several methods have been proposed to deal with this problem of integration of information. Probabilistic inverse problem theory allow combining the available information by characterizing (or sampling from) a posterior probability function that combines the information form the geostatistical model that describes realistic earth models (in form of a prior probability density), 
with information from data (in form of a likelihood function) (Tarantola, 2005). Using Monte Carlo sampling the posterior of any posterior probability can be sampled, as long as the prior model can be sampled, and the likelihood can be evaluated (Mosegaard and Tarantola, 1995; Hansen et al., 2008; Irving and Singha, 2010; Hansen et al., 2012; Cordua et al., 2012; Hansen et al., 2013). While such a Monte Carlo based approach can in principle deal with a large variety of very complex systems, its practical use is hampered by its very high computational demands.

Another approach is typically used in geostatistics, where available (geophysical) data are converted into 'soft data' about each individual model parameter. Soft data is a loosely defined term that typically refer to uncertainty and inequality constraints about about specific model parameters (Journel, 1986). Most all geostatistical simulation algorithms can make use of such 'soft' data (Remy et al., 2008; Mariethoz and Caers, 2014). However, challenges related to using current state of the art MPS simulation algorithms conditional to other geo-information has been considered widely in the literature with respect to ground water models He et al. (2014); Koch et al. (2014); Jørgensen et al. (2015); Biver et al. (2014); Høyer et al. (2015, 2017).

In the following the use of sequential simulation based MPS sampling methods will be considered for probabilistic data integration with independent uncertain conditional data, that may be available from other sources.

First, using notation from probabilistic data integration, we formulate precisely what is implicitly assumed about 'soft data' in most any MPS algorithm.

Through analysis of 3 reference models, with varying density of conditional/soft data, we demonstrate that a conventional implementation of sequential simulation based MPS simulation leads to simulations that fail to generate realizations (reservoir models) consistent with the available uncertain information (soft data).

Then, we suggest two novel approaches that allow considering the information in a more correct way using direct sampling (DS, Mariethoz et al., 2010), ENESIM (Guardiano and Srivastava, 1993), and SNESIM (Strebelle, 2000). The first use as preferential simulation path, where more informed model parameters are visited preferentially to less informed ones. The second approach involves using more than only co-located uncertain data, wihich is typically not done for most implementations of MPS. All examples are compared to those obtained by a general Monte Carlo based approach. 


\section{Data integration using conditional geostatistical simulation - Theory}

Consider that a model of the subsurface is parameterized into $M$ model parameters $\mathbf{m}=\left[m_{1}, m_{2}, m_{3}, \ldots, m_{M}\right]$. Say information is available about the model parameters $\mathbf{m}$ from $N$ independent sources $\mathbf{I}=\left[I_{1} 1, I_{2}, \ldots, I_{N}\right]$ through the probability densities $f_{I_{1}}(\mathbf{m}), f_{I 2}(\mathbf{m}), \ldots, f_{I_{N}}(\mathbf{m})$. Each probability distribution then represents a specific state of information. Tarantola and Valette (1982) and Tarantola (2005) demonstrate how these states of information can be combined through the conjunction of the states of information through

$$
\begin{aligned}
f_{\mathbf{I}}(\mathbf{m}) & =f_{I_{1}}(\mathbf{m}) \wedge f_{I_{2}}(\mathbf{m}) \wedge \ldots \wedge f I_{N}(\mathbf{m}) \\
& =\nu \mu(\mathbf{m})^{(1-N)} \prod_{i}^{N} f_{I_{i}}(\mathbf{m})
\end{aligned}
$$

where $\nu$ represents a normalizing constant, $\mu(\mathbf{m})$ represents the homogeneous probability distribution or the 'state of total ignorance' (Jaynes, 1968), and $\wedge$ is the operator for 'conjunction'. Conjunction of information, as expressed through (1), is derived from axioms similar to the axioms of formal logic on conjunction of propositions, and the Radon-Nikodym theorem from measure theory (Tarantola and Valette, 1982).

If a Cartesian coordinate system is used to parameterize $\mathbf{m}$, then the homogeneous probability density function becomes a constant $\mu(\mathbf{m})=k$ (Mosegaard and Tarantola, 2002), which is the case we will consider here. Then the problem of integrating information from independent sources into to one probability density $f_{\mathbf{I}}(\mathbf{m})$ is given by

$$
f_{\mathbf{I}}(\mathbf{m}) \propto \prod_{i}^{N} f_{I_{i}}(\mathbf{m}) .
$$

In the present context $\mathbf{m}$ reflects model parameters describing a reservoir model, and $I_{1}, I_{2}$, .. reflect different sources of information available (e.g. from expert information, well log data, training image and geophysical data).

Here, the special case is considered where all information available refers directly to the model parameters. The reason for this is two-fold: First, most (any) geostatistical simulation algorithms allow, in principle, to take such information into account as "soft" information (Mariethoz and Caers, 2014). Second, working with reservoir models, a lot of information about the 
model parameters of interest can be available in form of direct measurements from well logs, inverted well logs parameters, or indirectly from geophysical data inverted into information about the model parameters $\mathbf{m}$. Barfod et al. (2016) present a recent example of how to do this, by establishing an atlas (applicable in Denmark) that can be used to translate resistivity values (found through inversion of airborne EM data) into lithological/hydrological units with associated uncertainty.

Three types of information are available in a typical MPS based geostatistical data integration problem:

$I_{T I}$ Information from a training image. This can be information from outcrops, previous analysis, well log analysis, expert information which is quantified through a geostatistical model describing (spatial) codependence between model parameters.

$I_{\text {hard }}$ Hard data. Direct observation of one or more model parameters, without any associated uncertainty.

$I_{\text {soft }}$ Soft data. Direct observation of one or more model parameters, with an associated uncertainty.

In case the information has been obtained independently, such a geostatistical problem is equivalent to the problem of inferring information about $f_{\mathbf{I}}(\mathbf{m})$ given by

$$
f_{\mathbf{I}}(\mathbf{m}) \propto f_{I_{T I}}(\mathbf{m}) f_{I_{\text {hard }}}(\mathbf{m}) f_{I_{\text {soft }}}(\mathbf{m}) .
$$

Høyer et al. (2017) present one example of combining these three types of information into one stochastic model.

In principle there is no need to distinguish between hard and soft data, as both are simply data that provide information about the model parameters. So, a general geostatistical data integration problem can be formulated as

$$
f_{\mathbf{I}}(\mathbf{m}) \propto f_{I_{T I}}(\mathbf{m}) f_{I_{\text {data }}}(\mathbf{m}) .
$$

Spatially independent 'data'. For many geostatistical data integration problems, the information about each model parameter is assumed spatially independent, such that

$$
f_{I_{\text {data }}}(\mathbf{m})=\prod_{i=1}^{M} f_{I_{\text {data }}}\left(m_{i}\right) .
$$


From hereon, the term 'soft information' about the model parameters is defined through equation (5). The general data integration problem of equation (4) is then reduced to

$$
f_{\mathbf{I}}(\mathbf{m}) \propto f_{T I}(\mathbf{m}) f_{d a t a}(\mathbf{m})=f_{T I}(\mathbf{m}) \prod_{i=1}^{M} f_{d a t a}\left(m_{i}\right) .
$$

Equation (6) represent the probability distribution that most sequential simulation based MPS methods suggest to sample from, by combining information from a geostatistical model with 'hard' (certain) and 'soft' (uncertain) data. From hereon different methods, existing and new, will be discussed that allow sampling from equation (6).

\subsection{Markov chain Monte Carlo sampling of $f_{\mathbf{I}}(\mathbf{m}) \propto f_{T I}(\mathbf{m}) f_{\text {data }}(\mathbf{m})$}

Sampling methods such as the extended Metropolis sampler provides a general, but computationally expensive, approach for sampling the product of two (or more) probability densities, both in form of equation (4) (accounting for spatially dependent information on the model parameters) and (6) (assuming spatially independent information on the model parameters) Hansen et al. (2016a). Running the extended Metropolis algorithm consists of, in this case, sampling $f_{T I}(\mathbf{m})$ through a random walk, and accepting moving between proposed models based on acceptance criteria computed from the relative change in $f_{\text {data }}(\mathbf{m})$. Details on how to use the extended Metropolis sampler to sample from equation (4) and (6) can be found in e.g. Hansen et al. (2008, 2013, 2016a).

\subsection{Sequential simulation of $f_{\mathbf{I}}(\mathbf{m}) \propto f_{T I}(\mathbf{m}) f_{\text {data }}(\mathbf{m})$}

Sequential simulation (Alabert et al., 1989), also known as the conditional distribution method (Devroye, 1986), is commonly used in geostatistics to sample from $f_{I_{T I}}(\mathbf{m})$ and (conditional to data) $f_{\mathbf{I}}(\mathbf{m}) \propto f_{I_{T I}}(\mathbf{m}) f_{I_{\text {data }}}(\mathbf{m})$ as in equation (6). In brief, sequential simulation consists of sequentially visiting and simulating all model parameters, possibly in random order. At the location of each model parameter $m_{i}$, the value of $m_{i}$ is simulated (as $m_{i}^{*}$ ) conditional to all known information and all previously simulated model parameters, $\mathbf{m}_{c}$, as a realization from

$$
f_{\mathbf{I}}\left(m_{i} \mid m_{1}^{*}, \ldots, m_{i-1}^{*}\right)=f_{\mathbf{I}}\left(m_{i} \mid \mathbf{m}_{c}\right)=f_{T I}\left(m_{i} \mid \mathbf{m}_{c}\right) f_{\text {data }}(\mathbf{m})
$$


In case the available data are spatially independent, as in equation (6), the conditional distribution in equation (7) becomes

$$
f_{\mathbf{I}}\left(m_{i} \mid \mathbf{m}_{c}\right) \approx f_{T I}\left(m_{i} \mid \mathbf{m}_{c}\right) \prod_{i=1}^{M} f_{d a t a}\left(m_{i}\right)
$$

Numerous methods based on sequential simulation has been developed in the geostatistical community that allow sampling from a wide variety of multiple-point statistical models inferred from a training image such as given by $f_{T I}(\mathbf{m})$ (Guardiano and Srivastava, 1993; Strebelle, 2000; Mariethoz et al., 2010; Straubhaar et al., 2011; Hansen et al., 2016b)) These methods differ in how the realization $m_{i}^{*}$ of the conditional distribution in equation (7) is generated. Most of these methods allow, to some degree, to take into account direct information about the model parameters, hard and soft. In the following the ENESIM, SNESIM and Direct Sampling (DS) methods will be considered.

\section{A synthetic example}

In order to analyze the use of conditional information with sequential simulation algorithms based on MPS, a synthetic case study is designed. Figure 1a shows a training image (from Strebelle (2000), used to define $f_{I_{T I}}(\mathbf{m})$ ), consisting of pixels within (black) and outside (red) a channel structure, from which a reference model is generated as a realization in a $30 \times 30$ pixel grid, Figure 1b, using the ENESIM algorithm (Guardiano and Srivastava, 1993; Hansen et al., 2016b). The 25 closest previously simulated data are used to compute the conditional distribution at each step of the sequential simulation.

Simple smoothing of the reference realization in Figure $1 \mathrm{~b}$ is performed in order to obtain an exhaustive map of 'soft' data that quantifies the local probability of each pixel belonging to a channel structure through $f_{I_{d 1}}(\mathbf{m})$, Figure 2a. From this exhaustive set of soft data, a subset of 10 and 3 randomly chosen soft data points are considered as $f_{I_{d 2}}(\mathbf{m})$ and $f_{I_{d 3}}(\mathbf{m})$ and shown in Figures 2b-c.

The dense data set, $I_{d 1}$, mimic an exhaustive set of information, as obtained from for example inversion of a densely sampled electromagnetic data set, as considered extensively by Barfod et al. (2016). The two sparse data sets, $I_{d 2}$ and $I_{d 3}$, mimic information from well logs at different spatial density, 
as considered by for example Høyer et al. (2017). Note that the two sparse sets of soft data, quantified by $f_{I_{d 2}}(\mathbf{m})$ and $f_{I_{d 3}}(\mathbf{m})$, can both be regarded as an exhaustive set of soft data with a uniform distribution everywhere a soft data is not explicitly defined.

In the following existing and new methods for sampling $f_{I_{T I}, I_{d 1}}(\mathbf{m}), f_{I_{T I}, I_{d 2}}(\mathbf{m})$, and $f_{I_{T I}, I_{d 3}}(\mathbf{m})$, will be analyzed and compared.

[Figure 1 about here.]

[Figure 2 about here.]

\section{A 'reference' solution - sampling from $f_{I_{T I}, I_{d}}(\mathrm{~m})=f_{I_{T I}}(\mathrm{~m}) f_{I_{\text {data }}}(\mathrm{m})$}

The extended Metropolis algorithm is used to sample from $f_{\mathbf{I}}(\mathbf{m})$ considering the three soft data sets defined above. This provides a reference solution (in form of a sample from $f\left(\mathbf{m} \mid I_{T I}, I_{\text {data }}\right)$ ), to which other solutions can be compared. In practice, the ENESIM algorithm is used to generate realizations from $f\left(\mathbf{m} \mid I_{T I}\right)$ that are then accepted using the Metropolis acceptance criterion based on the soft data. In this way, 600 independent realizations have been obtained from $f_{I_{T I}, I_{d 1}}(\mathbf{m}), f_{I_{T I}, I_{d 2}}(\mathbf{m})$, and $f_{I_{T I}, I_{d 3}}(\mathbf{m})$, using the SIPPI Matlab toolbox (Hansen et al., 2013). The corresponding probability of locating a channel, obtained using the above described algorithm are shown in Figure 3a-c. These results will be used as a reference for comparison.

[Figure 3 about here.]

\section{Existing sequential simulation methods, using the Markov prop- erty}

Well known MPS algorithms such as ENESIM and SNESIM allow conditioning to uncertain data (Strebelle, 2000; Remy et al., 2008). In practice, most all MPS based sequential simulation algorithms use only co-located soft data (i.e. soft data located at the same position in space as the model parameter $m_{i}$ being simulated) when evaluating equation (8). The rest of the soft data are being ignored (see e.g.Strebelle (2000); Liu (2006); Remy et al. (2008)). In this case the marginal conditional probability being sampled during sequential simulation is reduced from equation (8) to

$$
f_{\mathbf{I}}\left(m_{i} \mid \mathbf{m}_{c}\right) \propto f_{T I}\left(m_{i} \mid \mathbf{m}_{c}\right) f_{\text {data }}\left(m_{i}\right)
$$


This assumption is similar to the Markov property assumed for sequential Gaussian co-simulation, as proposed by Almeida and Journel (1994). Therefore the approximation in equation (9) is referred to as using a Markov property to handle the soft data. Equation $((9))$ assumes that the source of the information from the training image, $f_{T I}\left(m_{i} \mid \mathbf{m}_{c}\right)$, and the 'soft' data, $f_{\text {data }}\left(m_{i}\right)$, are independent. If this is not the case, one can use e.g. the taumodel to explicitly model the dependence between the two types of available information (Journel, 2002; Krishnan, 2008). The amount of dependency is controlled by the tau factor. Estimation of a proper value of the tau factor, can in itself be a challenging task, and is not considered further here.

The complexity related to implementing an algorithm that samples from equation (9) depends on the choice of MPS algorithm. Below we briefly describe these differences for a number of widely used methods. We refer to Mariethoz and Caers (2014) for a general description of MPS algorithms.

\subsection{ENESIM and the Markov property}

Using ENESIM the full conditional distribution $f_{T I}\left(m_{i} \mid \mathbf{m}_{c}\right)$ is explicitly computed at each iteration by scanning the whole training image. Therefore evaluation of equation (9) is straightforward to implement.

\subsection{SNESIM type algorithms and the Markov property}

SNESIM (Strebelle, 2000), and related IMPALA (Straubhaar et al., 2011), type simulation algorithms scans the training image only once, for a number of predefined sets of conditional point patterns. The frequency of occurrence for each pattern is then stored in memory. At each iteration in the sequential simulation $f_{T I}\left(m_{i} \mid \mathbf{m}_{c}\right)$ is then obtained from memory, and hence evaluation of equation (9) straightforward.

However, SNESIM also makes use of so-called multiple-grids, that is needed to allow reproducing correlations over long distances, while at the same time reducing the memory requirements (Tran, 1994). This introduces a challenge when conditioning hard and soft data are available, as conditional data may not be available on a specific coarse grid being simulated. To remedy this, so-called re-location of hard data has been suggested. When simulating on a coarse grid, the closest hard data at finer grids are re-located to the coarse simulation grid as a hard data. Then conditional simulation is performed in the coarse grid. Finally after, simulation of the coarse grid the hard data values at the notes of the re-located data, are removed, and set as un-sampled. See details in Strebelle (2000); Remy et al. (2008). Here, 
re-location of the soft data has been implemented in the SNESIM implementation in MPSlib (Hansen et al., 2016b), in a manner similar to the approach used for hard data. Note that in case the uncertain/soft data are exhaustively available, no relocation is needed. Straubhaar and Malinverni (2014) propose an alternative approach for handling conditional data with multiple grids, that can lead to less artifacts.

\subsection{Handling co-located soft data using $D S$}

Using the DS algorithm $f_{T I}\left(m_{i} \mid \mathbf{m}_{c}\right)$ is never explicitly computed, instead a realization from $f_{T I}\left(m_{i} \mid \mathbf{m}_{c}\right)$ is obtained directly from the training image. This means the DS algorithm cannot take co-located soft data into account simply by evaluating equation (9).

Biver et al. (2014) and Straubhaar et al. (2016) suggest an approach that aims to reproduce the local proportions within a data neighborhood, as provided by $I_{\text {soft }}$ (for data of both point and volume support). In their approach uncertainty of the soft data is not taken into account explicitly as defined in equation (9). Instead we propose to use a simple application of the extended rejection sampler that allows the direct sampling algorithm to generate a realization of $f_{\mathbf{I}}\left(m_{i} \mid \mathbf{m}_{c}\right)=f_{T I}\left(m_{i} \mid \mathbf{m}_{c}\right) f_{\text {data }}\left(m_{i}\right)$, using the exact same conditions as ENESIM and SNESIM. Numerical implementation consists of replacing the step of scanning the training image for the first matching conditional data event $\mathbf{m}_{c}$, with the following algorithm

- Start loop

1. Obtain a realization, $m_{i}^{*}$, of $f_{T I}\left(m_{i} \mid \mathbf{m}_{c}\right)$ (by scanning the training image).

2. Accept $m_{i}^{*}$ as a realization of $f_{T I}\left(m_{i} \mid \mathbf{m}_{c}\right) f_{\text {data }}\left(m_{i}\right)$ with probability $P_{a c c}=\frac{f_{\text {data }}\left(m_{i}=m_{i}^{*}\right)}{\max \left(f_{\text {data }}\left(m_{i}\right)\right)}$.

- End loop (when $m_{i}^{*}$ is accepted) .

$\max \left(f_{\text {data }}\left(m_{i}\right)\right)$ is the maximum probability of any possible value of $m_{i}$. This will ensure that $m_{i}^{*}$ will be a realization of $f\left(m_{i} \mid \mathbf{m}_{c}\right) f_{\text {data }}\left(m_{i}\right)$ as given in equation (9). This rejection step has been implemented in the GENESIM algorithm in MPSlib (Hansen et al., 2016b), which is a generalized implementation of the ENESIM algorithm, in which the conditional distribution is based on any number $N_{c}$ of observed matches. If $N_{c}=1$, the GENESIM algorithm will in practice perform similar to the DS algorithm (Hansen et al., 
2016b). In the remainder, when we refer to the DS algorithm we use use the GENESIM algorithm with $N_{c}=1$.

\subsection{Conditional ENESIM/SNESIM/DS simulation using the Markov prop- erty}

Using the ENESIM algorithm and the Markov property for conditioning to 'soft' data, 600 independent realizations are generated and the corresponding probability of locating a channel computed. The results are shown in Figure 4 in case using a 'unilateral' (i.e., raster scan) path (top, a)-c)), and in case using a random path (bottom, d)-f)). Similar results obtained using SNESIM are shown in Figure 5. No results are shown using DS as they are essentially identical to those obtained using ENESIM in Figure 4.

[Figure 4 about here.]

[Figure 5 about here.]

Figure 4 reveals that the simulation results lack information as compared to the full solution (Figure 3). This is most severe in case soft data are sparse in which case little to no information from the soft data seems to have been taken into account (Figure $4 \mathrm{~b}-\mathrm{c}$ and $4 \mathrm{e}-\mathrm{f}$ ). So, while it is rather straightforward to account for uncertain information about the model parameters using the Markov property (as also stated by Straubhaar et al., 2016), it may not be a viable approach using either a sequential or random simulation path. Below we propose two alternative approaches to better account for the available uncertain/soft data.

\section{Suggestion 1: preferential simulation path}

It has long been known that the choice of simulation path affects the realizations generated using sequential simulation (Strebelle, 2000; Liu and Journel, 2004; Daly, 2005; Mariethoz and Renard, 2010; Daly, 2005). One problem of using either the unilateral or random path with the Markov property as considered above, is that information from highly informed model parameters located very close to a model parameter, for which the conditional distribution is computed, is disregarded. Consider two direct observations $f\left(m_{i}=1\right)=0.999$ and $f\left(m_{j}=1\right)=1$ (which implies $f\left(m_{i}=0\right)=0.001$ and 
$f\left(m_{j}=0\right)=0$, as the training image only allows $\mathrm{k}=2$ possible outcomes $)$. The entropy

$$
E(f(m))=-\sum_{k} f\left(m=m^{k}\right) \log _{2}\left(f\left(m=m^{k}\right)\right),
$$

is a measure of uncertainty of the information provided by $f(m)$ (Reza, 1961). With $\mathrm{k}=2$ possible outcomes, the maximum entropy is given by $E_{\text {max }}(f(m))=1$. A base of 2 is used for the logarithm in equation (10), which is a natural choice with $\mathrm{k}=2$ possible outcomes. A base of $\mathrm{k}$, would be a natural choice for a training images with $\mathrm{k}$ possible outcomes. A simple measure of the 'certainty' of the information provided by $f(m)$ can then be formulated as

$$
C(f(m))=1-\frac{E(f(m))}{E_{\max }}
$$

This leads to $C\left(f\left(m_{i}\right)\right)=0.99$ and $C\left(f\left(m_{j}\right)\right)=1$. Thus, these two types of information provide almost the same information. However, in a typical implementation of an MPS algorithm (as discussed above) $f\left(m_{j}=1\right)=1$ is treated as hard data, and the value of $m_{j}$ is fixed at $m_{j}^{*}=1$ prior to simulation. This means that $m_{j}^{*}=1$ will be used as conditional data in any subsequent step of the sequential simulation algorithm.

The information provided by $f\left(m_{i}=1\right)=0.99$ will however be treated as uncertain/soft data, and will (using the Markov property) only come into use when the simulation algorithm visits $m_{i}$, when a realization of $f\left(m_{i} \mid \mathbf{m}_{c}\right)$ has to be generated. Depending on the choice of random path this can happen early or late in the simulation process. If it happens early, then the information in $f\left(m_{i}=1\right)=0.99$ will affect the simulated value of relatively many model parameters. If it happens late in the process the information will only affect relatively few model parameters. Due to the use of the Markov property, the amount of information used for a given model parameter is closely related to the choice of random path. This is the reason for the relatively poor conditioning to the soft data obtained using sequential simulation with the Markov Property, using both a unilateral and random path as seen in Figures 4-5.

To remedy some of these problems the use of a preferential random path is suggested, where model parameters with soft data with high information content is visited preferentially to soft data with lower information content.

In practice the preferential path can be computed prior to running the sequential simulation algorithm. First, the entropy $E\left(f_{\text {data }}\left(m_{i}\right)\right)$ is computed 
for all soft data. Then, a pseudo random path is given by ordering all the model parameters in ascending order by order $i$ given by

$$
\operatorname{order}_{i}=r_{i}-1+I_{f a c} C(f(m)) \text {, }
$$

where $r_{i}$ is a random number between 0 and $1 . I_{f a c}$ is a factor that controls the 'randomness' associated to the information content. If $I_{f a c}=0$ all model parameters with soft data are visited at random (in no specific order), before model parameters with no soft data are visited. When $I_{f a c}$ is high then locations with soft data are visited in order of decreasing information content. In the following $I_{f a c}=4$ is used.

\subsection{Conditional ENESIM/SNESIM/DS simulation using the Markov prop- erty and the preferential path}

Figures 6,7 , and 8 show the probability of locating a channel conditional to the three data sets, based on 600 realizations generated by ENESIM, DS, and SNESIM using a preferential path. If $P_{m c m c}($ channel $)$ and $P($ channel $)$ refer to the posterior probability of locating a channel in each pixel using the reference MCMC approach and a specific choice of simulation, then Tables 3-1 summarize the relative difference in L2-norm as $L_{2}\left(P_{m c m c}(\right.$ channel $)-$ $P($ channel $)) / L_{2}\left(P_{m c m c}(\right.$ channel $\left.)\right)$, for different simulation choices and choice of simulation algorithm. A number close to 0 suggests that simulation results (in form of the posterior probability of locating a channel) is very close to the results obtained using the reference McMC approach, Figure 3, while a higher number will refer to less similarity. From hereon we refer to this quantity as the 'relative L2 norm'.

\subsubsection{ENESIM}

Using ENESIM with a preferential path conditional to $I_{d 1}$, it is clear that not as much information is extracted from the uncertain data, Figure 6a, as is the case using full Monte Carlo sampling, Figure 3a. This difference is due the fact that the Markov property is not used as part of the Monte Carlo sampling, which will lead to better resolved channel structures. However, significantly more information is extracted than when using an unilateral or random simulation path, see Figures 4a and 4d. Table 1 also shows a significant drop in the relative L2-norm using the preferential path (0.43 vs 0.69 using a random path).

In the case of sparse data $\left(I_{d 2}\right.$ and $\left.I_{d 3}\right)$ the use of a preferential path provides results, Figure $6 \mathrm{~b}-\mathrm{c}$, that are close to indistinguishable from the full 
non-Markov solution, obtained using Monte Carlo sampling, Figure 3b-c., with a corresponding small L2 norm, Table 1.

[Figure 6 about here.]

\subsection{2. $D S$}

The results obtained using DS, Figure 7, are similar to the results obtained using the ENESIM algorithm, Figure 6, and quantified in Table 2, illustrating that the use of the rejection sampler with DS works as intended.

[Figure 7 about here.]

\subsubsection{SNESIM}

Comparing Figure 5 to 8 it is evident that the use of a soft data relocation and a preferential path with SNESIM allow much better reproduction of uncertain data. However, some effects of using multiple grids and re-location persist, which is the reason of the relative high relative L2 norm of 0.23 using SNESIM compared to 0.09 using ENESIM and DS in case conditioning to $I_{d 3}$, see Tables 1-3.

One simple approach to remedy some of the effects of re-location of soft (and hard) data, is to make use of ENESIM type algorithms to perform the simulation on coarser grids, as suggested by Strebelle (2000) to avoid problems related to hard-data relocation. Another approach could be to consider applying the approach proposed by Straubhaar and Malinverni (2014) also to soft/uncertain data, to avoid artifacts caused by the use of multiple grids.

[Figure 8 about here.]

Tables 1-3 highlights that in general the use of the preferential path, with the Markov assumption considering only colocated data, significantly reduces the relative L2 norm. Further Tables 1-3 suggest the difference in simulation time using the preferential path compared to using the random path is small.

The preferential path emulates what has been done in practice since the first simulation algorithms were developed. If 'hard' information is available, i.e. certain information about the model parameters, then these model parameters will be visited before other model parameters using the preferential path. This is equivalent to simply assigning the hard data to the corresponding model parameters prior to starting the simulation. It is also related to 
simulating model parameters with soft information prior to other data, as proposed by Soares et al. (2016) in case using Gaussian direct sequential simulation.

Liu and Journel (2004) also suggest to choose the random path guided by the information content. Unlike the present work, where the path is guided by the information content of the soft data, they suggest to guide the path based on the conditional information from the training image, i.e. from $f_{T I}\left(m_{i} \mid \mathbf{m}_{c}\right)$. They demonstrate that such a path better reproduces large scale connected structures, compared to using a random simulation path.

\section{Suggestion 2: Avoiding the Markov property}

The Markov property can in principle be avoided entirely, to allow considering more than just co-located soft information, while still using the sequential simulation approach, and using a fully random path.

\subsection{DS conditional to non-colocated soft data}

The extended rejection sampler used above to allow the DS algorithm to condition to co-located uncertain/soft data, can be generalized to account for, in principle, all soft data, without the need for the Markov property. A sample of equation (6) can be obtained at each iteration of the sequential simulation algorithm using the extended rejection sampler as follows:

- Start loop

1. Obtain a realization, $m_{i}^{*}$, of $f_{T I}\left(m_{i} \mid \mathbf{m}_{c}\right)$.

2. Accept $m_{i}^{*}$ as a realization of $f_{T I}\left(m_{i} \mid \mathbf{m}_{c}\right) \prod_{i=1}^{M} f_{\text {data }}\left(m_{i}\right)$ with probability

$$
P_{a c c}=\frac{\prod_{i=1}^{N_{S}} f_{\text {data }}\left(m_{i}=m_{i}^{*}\right)}{\prod_{i=1}^{N_{s}} \max \left(f_{\text {data }}\left(m_{i}\right)\right)}
$$

- Continue loop (until $m_{i}^{*}$ is accepted).

$N_{s}$ refers to the closest $N_{s}$ soft/uncertain data. In case $N_{s}=\infty$, the above will sample from full probability density given in equation (6), without the Markov assumption. Hence, results should be comparable to using the Monte Carlo based sampling approach.

In practice, due to both CPU requirements and the limited size of the training image, $N_{s}$ can be chosen to use limited set of conditional soft data, while providing simulation results similar to using a full neighborhood, using much less computational power. 
Conditional simulation to soft data, without the preferential path. When conditioning to non-colocated soft data, the use of the preferential path should, in principle, no longer be needed in order to condition to soft/uncertain data. Figure 9 shows the probability of locating a channel in case using a random path, and the 3 closest soft/uncertain data using DS type simulation using the rejection sampling approach described above.

In general the resolution is better than using a unilateral or random with the Markov property, but worse than using a preferential path and the Markov property (see e.g. Table 2)

This is due to conditioning to soft data becoming more difficult if a lot of model parameters are visited, and hence simulated, prior to visiting the location of the soft data. In this case the 'hard' simulated data will take precedence over the soft data, unless a non-perfect match to the hard data is allowed. This is one reason why the use of the preferential path may be useful even when conditioning to non-colocated soft data.

Conditional simulation to soft data, with the preferential path.. Another reason to use the preferential path in this case is that it can lead to a computationally more efficient simulation algorithm. Using a random path, one will have to evaluate the rejection sampler described above, at all iterations until all model parameters with soft data have been simulated. If using a preferential random path, one need only evaluate the rejection sampling step above, until all soft data has been evaluated. Thus, only for the first 3 and 11 iterations considering $I_{d 3}$ and $I_{d 2}$.

Figure 10 shows results obtained running the DS algorithm to generate 600 independent realizations, using $N_{s}=1$ (top), $N_{s}=3$ (middle), and $N_{s}=11$ without the Markov property, with a preferential path. Table 2 shows the corresponding relative L2-norm and simulation time.

For the most sparse data set, $I_{d 3}$, a subtle difference can be identified comparing figure 10c) $\left(N_{s}=1\right)$ and 10f $)\left(N_{s}=3\right)$, leading to a slightly smaller relative L2 norm. Considering $N_{s}=3$, the probability of locating a channel is slightly larger than when using $N_{s}=1$. In general, there is little to no difference using $N_{s}=3$ or $N_{s}=11$ conditioning to sparse soft data, $I_{d 2}$ and $I_{d 3}$.

It is also clear that when conditioning to the exhaustive soft data set, $I_{d 1}$, the amount of information extracted from the soft data (as quantified in Table 2), increases as the number of conditioning soft data increases, Figure $10 \mathrm{a}, \mathrm{d}, \mathrm{g}$. For this conditional data set, the best result (i.e. that best resemble 
the reference solution) is obtained using 11 conditional data, Figure 10g.

This algorithm, as any rejection algorithm, will only be feasible if the number of conditioning soft data is small. Alternatively one can make use of only a limited number of the closest soft data, to allow a better use of the soft data, while limiting the computational needs.

Note in Table 2 that when using a random simulation path, and noncolocated soft data, results in a significant increase in simulation times (a factor of 1-8) when using more non-located soft data as compared to only one soft data. Using the preferential path the simulation times is only a few percent larger using 25 conditional soft data, as opposed to 1 conditional soft data, in the case of conditioning to e.g $I_{d 2}$.

\subsection{ENESIM/GENESIM conditional to non-colocated soft data}

The ENESIM/GENESIM algorithm can be also generalized to sample conditionally to non-colocated soft data. In this case the whole (using ENESIM) or a limited random part (using GENESIM) of the training image is scanned at each iteration. For each match of a hard data, the specific value of the centered node in the training image, is associated with the (soft) probability $\prod_{i=1}^{N_{s}}\left(f_{\text {data }}\left(m_{i \mid j}\right)\right) . \quad j$ is the position in the training image and $m_{i \mid j}$ refer to the value of the location of the soft data relative to the current location in the training image. Conditioning to non-colocated soft data as described here have been implemented in the MPSlib codes in the GENESIM algorithm, Hansen et al. (2016b).

Simulation times and relative L2 norms using GENESIM type simulation are, for reference, presented in Table 1, for the same conditional data sets considered by DS in Table 2. Even though the handling of soft data in DS and ENESIM type simulation is quite different, the main difference between the two algorithms are with respect to simulation times, which is expected. The GENESIM algorithm can be used to scan only a limited set conditional evenets, which is much faster than using ENESIM that scans the entire training image at each iteration.

\subsection{SNESIM conditional to non-colocated soft data}

While SNESIM can in principle also be generalized to account for noncolocated soft data, problems related to re-location persist, and search times scanning the search tree will become large. Therefore, we do no pursue this approach further, and leave this for potential future research. 
[Figure 9 about here.]

[Figure 10 about here.]

\section{Conclusion}

MPS based sequential simulation algorithms allow for a computationally efficient approach to the problem of integration of probabilistic information from different sources. However, the traditionally used Markov property, using only co-located uncertain soft data, leads to realizations that do not fully take into account the information of the soft data. The problem is most severe when sequential simulation is performed with soft information available at sparse locations. Two methods have been proposed that allow taking soft data properly into account.

First, a simulation path preferential to 1D marginal entropy/information content of soft data has been proposed. This allows much better handling of especially scattered soft data. The preferential path is trivial to use with the ENESIM algorithm. Using a simple rejection step to account for soft data, it can be easily implemented with the DS algorithm. It is straightforward to use with the SNESIM algorithm, but re-location of soft data is suggested due to the use of multiple grids.

Second, an approach is suggested that avoid the Markov-property, such that non co-located soft data can be considered, that can be used with any of the ENESIM and DS algorithms. Combined with using a preferential path this leads to a conditional simulation algorithm that properly conditions to the soft data, while at the same time being computationally much more viable than using McMC sampling methods.

\section{Acknowledgments}

This research has been funded by two projects (113-2013-1,53-2014-3) funded partly by the Danish High Technology Foundation. MPS simulation codes and examples are available at https://github.com/ergosimulation/mpslib/.

\section{References}

Alabert, F., et al., 1989. Non-gaussian data expansion in the earth sciences. Terra Nova 1 (2), 123-134. 
Almeida, J. S., Journel, A. G., 1994. Joint simulation of multiple variables with a markov-type coregionalization model. Mathematical Geology 26 (5), $465-588$.

Barfod, A., Mller, I., Christiansen, A., 2016. Compiling a national resistivity atlas of denmark based on airborne and ground-based transient electromagnetic data. Journal of Applied Geophysics.

Biver, P., Mariethoz, G., haar, J., Chugunova, T., Renard, P., 2014. Handling soft probabilities in multiple point statistics simulation. In: Mathematics of Planet Earth. Springer, pp. 69-72.

Cordua, K. S., Hansen, T. M., Mosegaard, K., 2012. Monte Carlo full waveform inversion of crosshole GPR data using multiple-point geostatistical a priori information. Geophysics 77, H19-H31.

Daly, C., 2005. Higher order models using entropy, markov random fields and sequential simulation. geostatistics Banff 2004, 215-224.

Deutsch, C., Journel, A., 1992. GSLIB: Geostatistical Software Library and User's Guide. Oxford University Press.

Devroye, L., 1986. Sample-based non-uniform random variate generation. In: Proceedings of the 18 th conference on Winter simulation. ACM, pp. 260-265.

Guardiano, F. B., Srivastava, R. M., 1993. Multivariate geostatistics: beyond bivariate moments. In: Geostatistics Troia 92. Springer, pp. 133-144.

Hansen, T., Cordua, K., Looms, M., Mosegaard, K., 2013. SIPPI: a Matlab toolbox for sampling the solution to inverse problems with complex prior information: Part 1, methodology. Computers \& Geosciences 52, 470-480.

Hansen, T., Cordua, K., Zunino, A., Mosegaard, K., 2016a. Probabilistic integration of geo-information. In: Moorekamp, M. (Ed.), Integrated Imaging of the Earth: Theory and Applications. AGU, pp. 93-116.

Hansen, T. M., Cordua, K. C., Mosegaard, K., 2012. Inverse problems with non-trivial priors - efficient solution through sequential Gibbs sampling. Computational Geosciences 16 (3), 593-611. 
Hansen, T. M., Mosegaard, K., Cordua, K. C., 2008. Using geostatistics to describe complex a priori information for inverse problems. In: Ortiz, J. M., Emery, X. (Eds.), VIII International Geostatistics Congress. Vol. 1. Mining Engineering Department, University of Chile, pp. 329-338.

Hansen, T. M., Vu, L. T., Bach, T., 2016b. MPSLIB: A C ++ class for sequential simulation of multiple-point statistical models. Software $\mathrm{X} 5$, $127-133$.

He, X., Sonnenborg, T., Jørgensen, F., Jensen, K. H., 2014. The effect of training image and secondary data integration with multiple-point geostatistics in groundwater modelling. Hydrology and Earth System Sciences 18 (8), 2943-2954.

Høyer, A.-S., Jørgensen, F., Foged, N., He, X., Christiansen, A., 2015. Threedimensional geological modelling of aem resistivity dataa comparison of three methods. Journal of Applied Geophysics 115, 65-78.

Høyer, A.-s., Vignoli, G., Hansen, T. M., Keefer, D. A., Jørgensen, F., et al., 2017. Multiple-point statistical simulation for hydrogeological models: 3D training image development and conditioning strategies. Hydrology and Earth System Sciences Discussions 21, 6069-6089.

Irving, J., Singha, K., 2010. Stochastic inversion of tracer test and electrical geophysical data to estimate hydraulic conductivities. Water Resources Research 46.

Jaynes, E. T., 1968. Prior probabilities. IEEE Transactions on systems science and cybernetics 4 (3), 227-241.

Jørgensen, F., Høyer, A.-S., Sandersen, P. B., He, X., Foged, N., 2015. Combining 3D geological modelling techniques to address variations in geology, data type and density-an example from southern denmark. Computers \& Geosciences 81, 53-63.

Journel, A., 1986. Constrained interpolation and qualitative information the soft kriging approach. Mathematical Geology 18 (3), 269-286.

Journel, A., 2002. Combining knowledge from diverse sources: An alternative to traditional data independence hypotheses. Mathematical geology 34 (5), $573-596$. 
Koch, J., He, X., Jensen, K. H., Refsgaard, J. C., 2014. Challenges in conditioning a stochastic geological model of a heterogeneous glacial aquifer to a comprehensive soft data set. Hydrology and Earth System Sciences 18 (8), 2907-2923.

Krishnan, S., 2008. The tau model for data redundancy and information combination in earth sciences: Theory and application. Mathematical Geosciences 40 (6), 705 .

Liu, Y., 2006. Using the snesim program for multiple-point statistical simulation. Computers \& Geosciences 32 (10), 1544-1563.

Liu, Y., Journel, A., 2004. Improving sequential simulation with a structured path guided by information content. Mathematical Geology 36 (8), 945964.

Mariethoz, G., Kelly, B. F., 2011. Modeling complex geological structures with elementary training images and transform-invariant distances. Water Resources Research 47 (7).

Mariethoz, G., Renard, P., 2010. Reconstruction of incomplete data sets or images using direct sampling. Mathematical Geosciences 42 (3), 245-268.

Mariethoz, G., Renard, P., Straubhaar, J., 2010. The direct sampling method to perform multiple-point geostatistical simulations. Water Resources Research 46 (11).

Mariethoz, P., Caers, P., 2014. Multiple-point Geostatistics: Stochastic Modeling with Training Images. Wiley.

Mosegaard, K., Tarantola, A., 1995. Monte carlo sampling of solutions to inverse problems. J. geophys. Res 100 (B7), 12431-12447.

Mosegaard, K., Tarantola, A., 2002. Probabilistic approach to inverse problems. International Geophysics 81, 237-265.

Remy, N., Boucher, A., Wu, J., 2008. Applied Geostatistics with SGeMS: A User's Guide. Cambridge University Press.

Renard, P., Straubhaar, J., Caers, J., Mariethoz, G., 2011. Conditioning facies simulations with connectivity data. Mathematical Geosciences 43 (8), 879-903. 
Reza, F. M., 1961. An introduction to information theory. Courier Corporation.

Soares, A., Nunes, R., Azevedo, L., 2016. Integration of uncertain data in geostatistical modelling. Mathematical Geosciences, 1-21.

Straubhaar, J., Malinverni, D., 2014. Addressing conditioning data in multiple-point statistics simulation algorithms based on a multiple grid approach. Mathematical Geosciences 46 (2), 187-204.

Straubhaar, J., Renard, P., Mariethoz, G., 2016. Conditioning multiple-point statistics simulations to block data. Spatial Statistics 16, 53-71.

Straubhaar, J., Renard, P., Mariethoz, G., Froidevaux, R., Besson, O., 2011. An improved parallel multiple-point algorithm using a list approach. Mathematical Geosciences 43 (3), 305-328.

Strebelle, S., 2000. Sequential simulation drawing structures from training images. Ph.D. thesis, Stanford University.

Tahmasebi, P., Sahimi, M., Caers, J., 2014. Ms-ccsim: accelerating patternbased geostatistical simulation of categorical variables using a multi-scale search in fourier space. Computers \& Geosciences 67, 75-88.

Tarantola, A., 2005. Inverse problem theory and methods for model parameter estimation. SIAM.

Tarantola, A., Valette, B., 1982. Inverse problems= quest for information. J. geophys 50 (3), 150-170.

Toftaker, H., Tjelmeland, H., 2013. Construction of binary multi-grid markov random field prior models from training images. Mathematical Geosciences 45 (4), 383-409.

Tran, T. T., 1994. Improving variogram reproduction on dense simulation grids. Computers \& Geosciences 20 (7-8), 1161-1168.

Zinn, B., Harvey, C. F., 2003. When good statistical models of aquifer heterogeneity go bad: A comparison of flow, dispersion, and mass transfer in connected and multivariate gaussian hydraulic conductivity fields. Water Resources Research 39 (3). 
[Table 1 about here.]

[Table 2 about here.]

[Table 3 about here.] 


\section{List of Figures}

1 a) Training image. b) Reference realization. Pixel color refer to inside (black) and outside (red) a channel. . . . . . . . . . . 25

2 Soft data. a) Exhaustive, 900 soft data $f_{I_{d 1}}(\mathbf{m})$, b) 10 soft data, $f_{I_{d 2}}(\mathbf{m})$, and c) 3 soft data, $f_{I_{d 3}}(\mathbf{m}) \ldots \ldots 26$

3 Posterior probability of locating a channel, $P\left(m_{i}=1 \mid I_{T I}, I_{d}\right)$, obtained using the extended Metropolis sampler, conditional to the three sets of soft data a) Exhaustive, d1, b) 10 random soft data, $d 2$, and c) 3 random soft data, $d 3 . \ldots . . . . . .27$

4 Posterior probability of locating a channel using the ENESIM algorithm with a top) unilateral and bottom) random path, conditional to a),d) $d 1, \mathrm{~b}), \mathrm{e}) d 2$, and c),f) $d 3$. Compare to Figure 3. . . . . . . . . . . . . . . . . . . 28

5 Posterior probability of locating a channel using the SNESIM algorithm with a top) unilateral, and bottom) random path, conditional to a),d) $d 1$, b),e) $d 2$, and c),f) $d 3$. . . . . . . . 29

6 Posterior probability of locating a channel using the ENESIM algorithm with a preferential path, conditional to a) $d 1, \mathrm{~b}$ ) $d 2$, and c) $d 3$. Compare to Figure 4 and the 'full' solution in Figure 3. . . . . . . . . . . . . . . . . . . . 30

7 Posterior probability of locating a channel using the DS algorithm with a preferential path, conditional to a) $d 1$, b) $d 2$, and

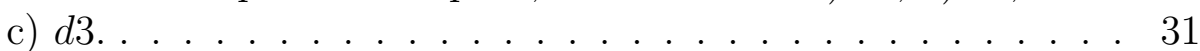

8 Posterior probability of locating a channel using the SNESIM algorithm with a preferential path, conditional to a) $d 1$, b) $d 2$, and c) d3. . . . . . . . . . . . . . . . . . . . 32

9 Posterior probability of locating a channel using the DS algorithm using the 3 closest 'soft' data and the 25 closest previously simulated data, with a random path, conditional to a)

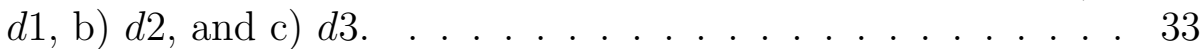

10 Posterior probability of locating a channel using the DS algorithm using the closest 1 (a,b,c), 3 (d,e,f), and 11 (g,h,i) 'soft'/uncertain data and the 25 closest previously simulated data, with a preferential path, conditional to $d 1$ (a,d,f), $d 2$ $(\mathrm{b}, \mathrm{e}, \mathrm{g})$, and $d 3 .(\mathrm{c}, \mathrm{f}, \mathrm{h}) \ldots \ldots . \ldots . \ldots . \ldots 34$ 

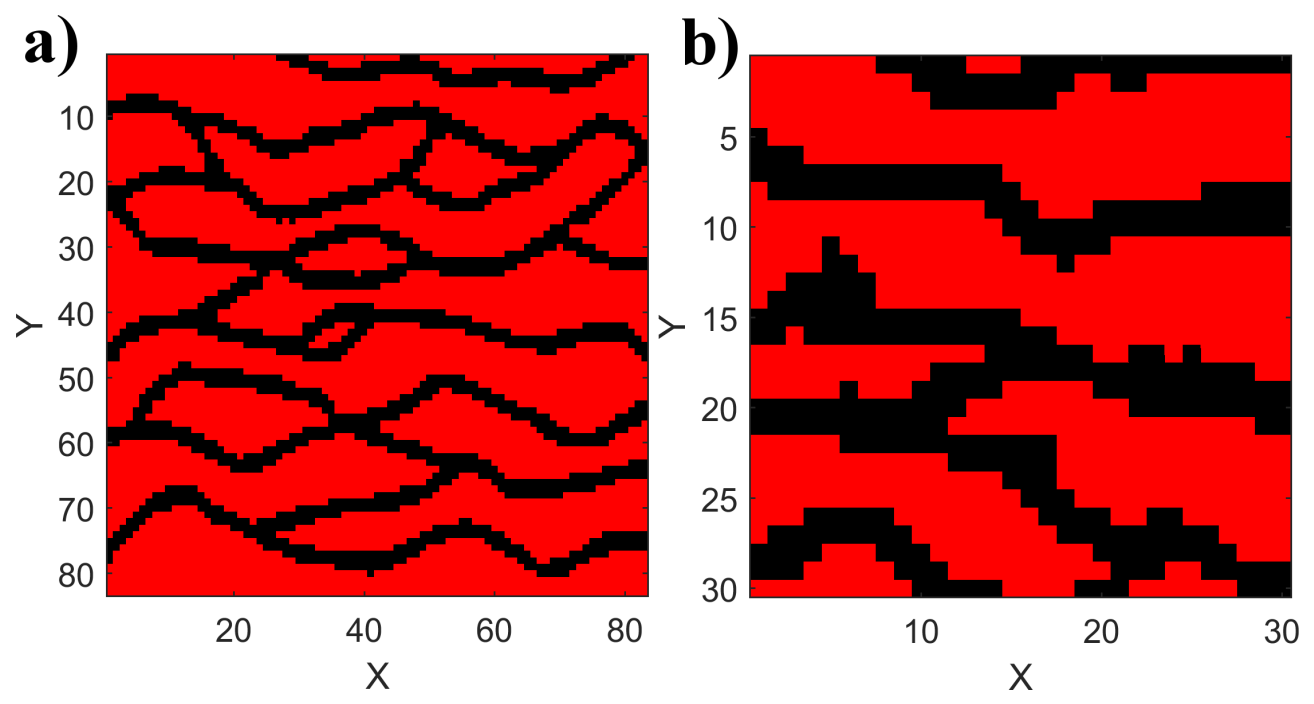

Figure 1: a) Training image. b) Reference realization. Pixel color refer to inside (black) and outside (red) a channel. 

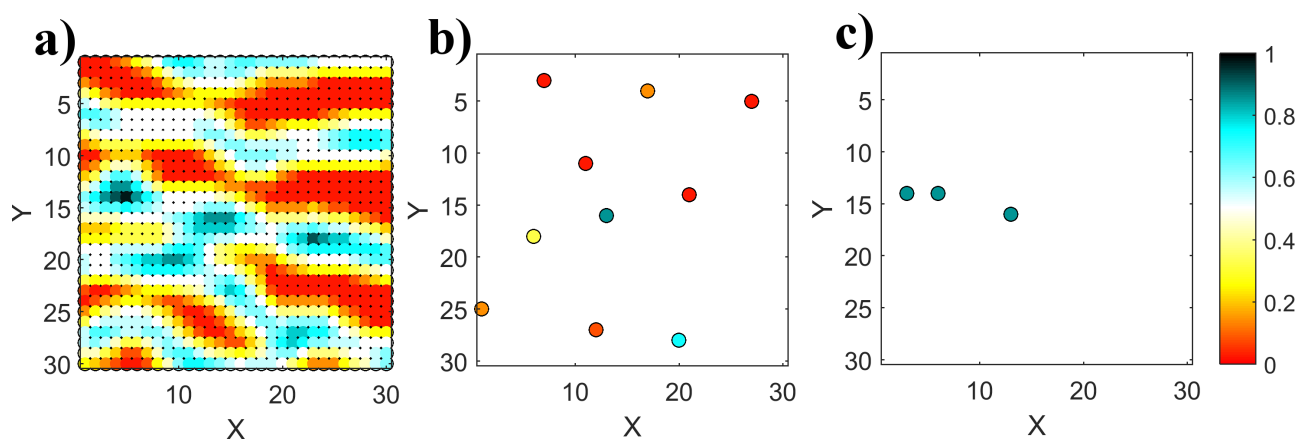

Figure 2: Soft data. a) Exhaustive, 900 soft data $f_{I_{d 1}}(\mathbf{m})$, b) 10 soft data, $f_{I_{d 2}}(\mathbf{m})$, and c) 3 soft data, $f_{I_{d 3}}(\mathbf{m})$ 

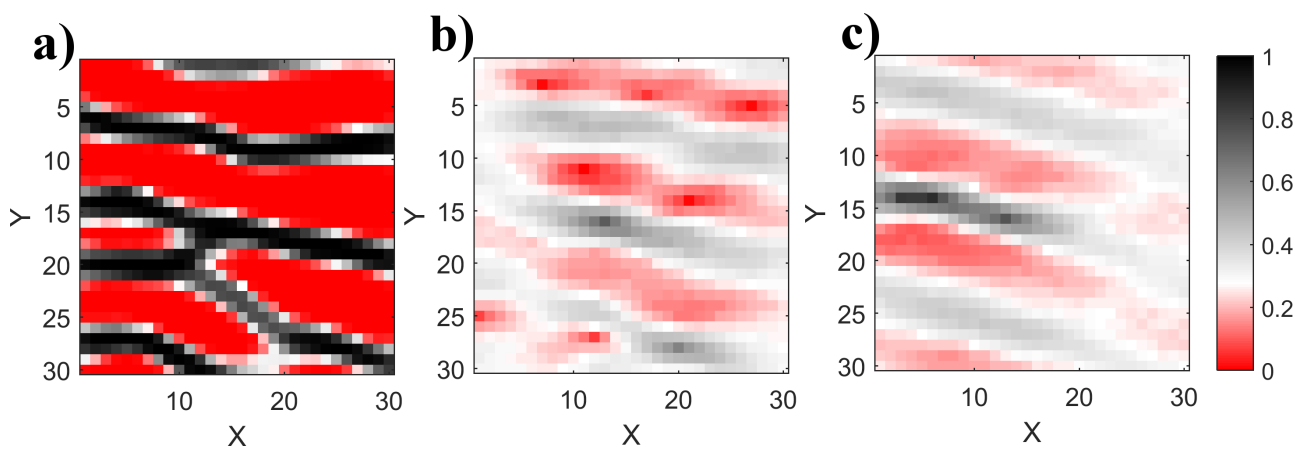

Figure 3: Posterior probability of locating a channel, $P\left(m_{i}=1 \mid I_{T I}, I_{d}\right)$, obtained using the extended Metropolis sampler, conditional to the three sets of soft data a) Exhaustive, $d 1$, b) 10 random soft data, $d 2$, and c) 3 random soft data, $d 3$. 

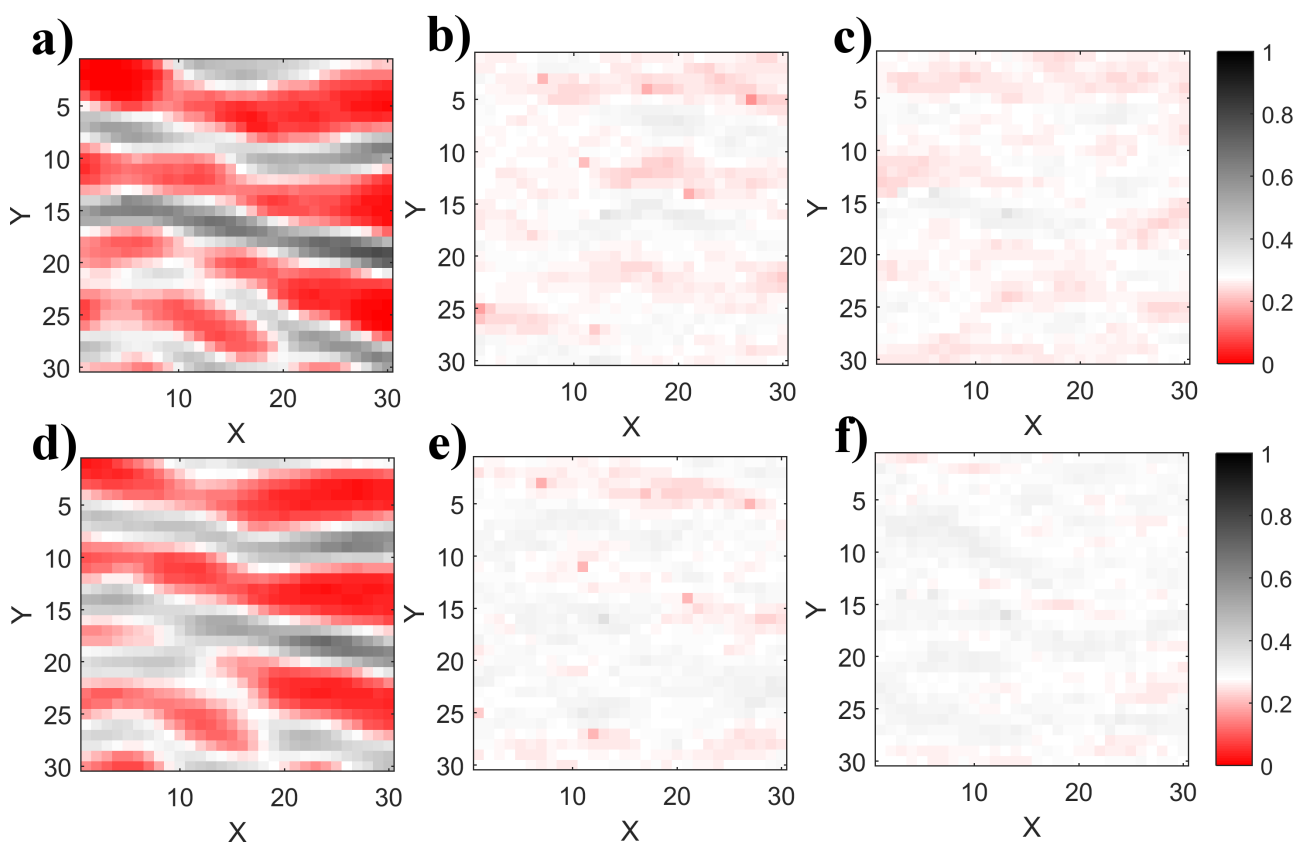

Figure 4: Posterior probability of locating a channel using the ENESIM algorithm with a top) unilateral and bottom) random path, conditional to a),d) $d 1$, b),e) $d 2$, and c),f) $d 3$. Compare to Figure 3. 

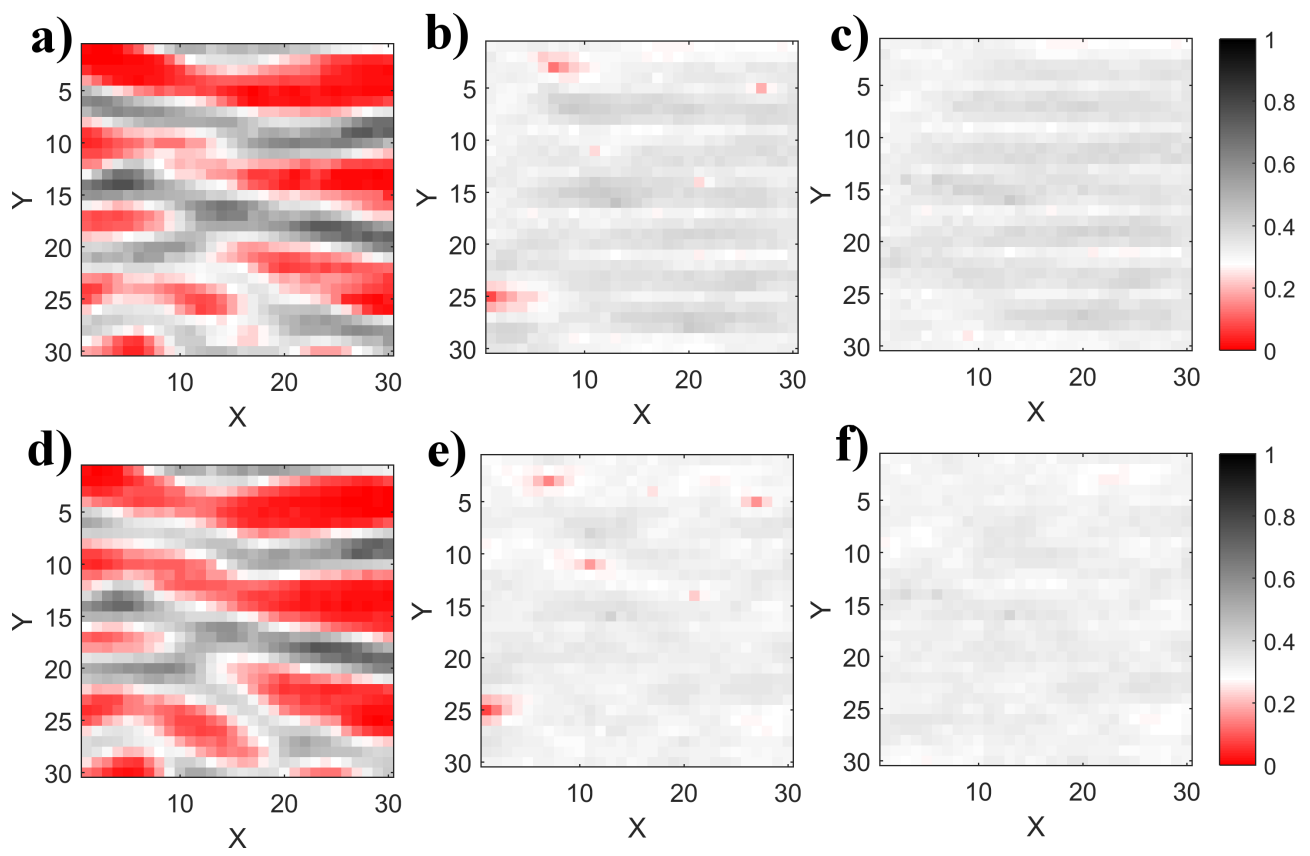

Figure 5: Posterior probability of locating a channel using the SNESIM algorithm with a top) unilateral, and bottom) random path, conditional to a),d) $d 1$, b),e) $d 2$, and c),f) $d 3$. 

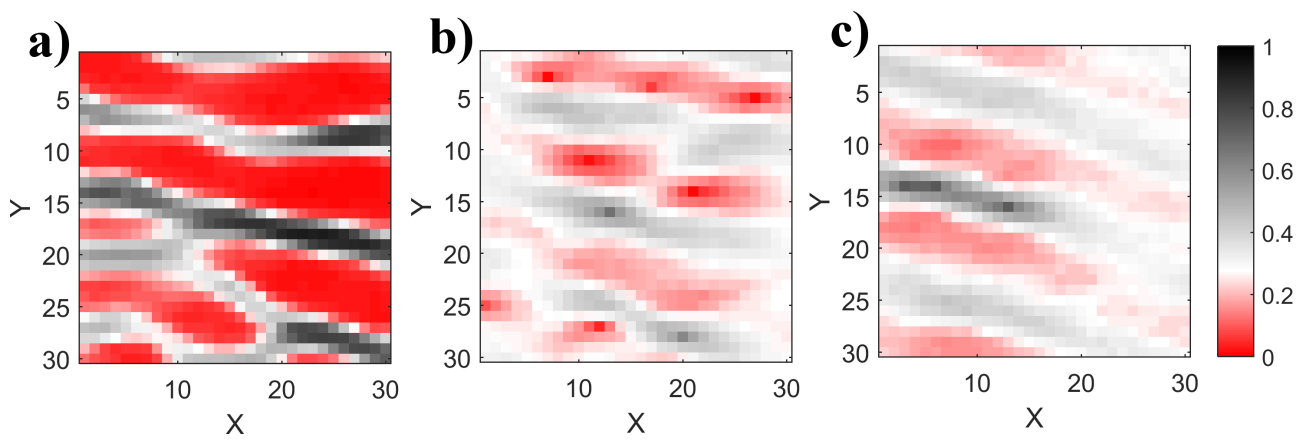

Figure 6: Posterior probability of locating a channel using the ENESIM algorithm with a preferential path, conditional to a) $d 1, \mathrm{~b}) d 2$, and c) $d 3$. Compare to Figure 4 and the 'full' solution in Figure 3. 

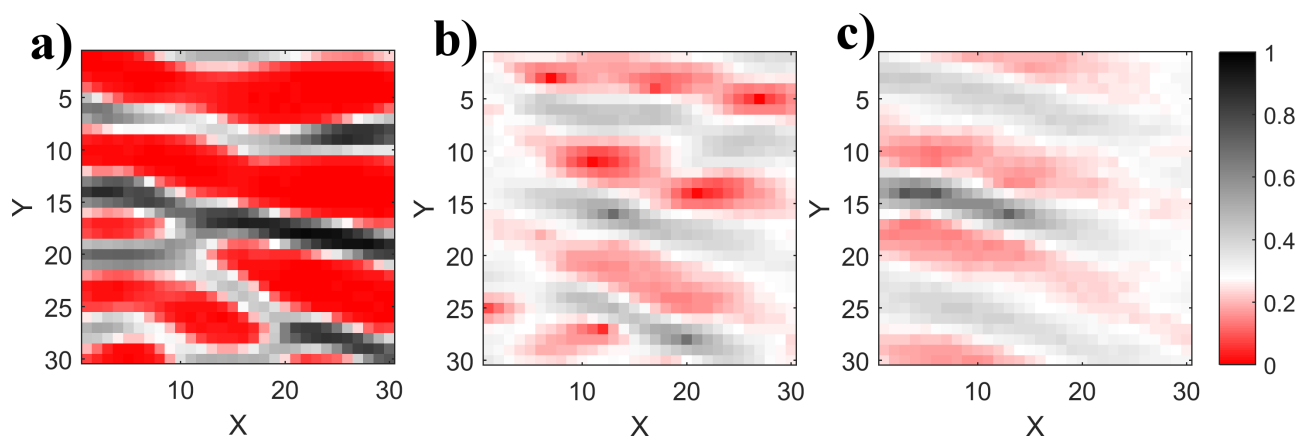

Figure 7: Posterior probability of locating a channel using the DS algorithm with a preferential path, conditional to a) $d 1$, b) $d 2$, and c) $d 3$. 

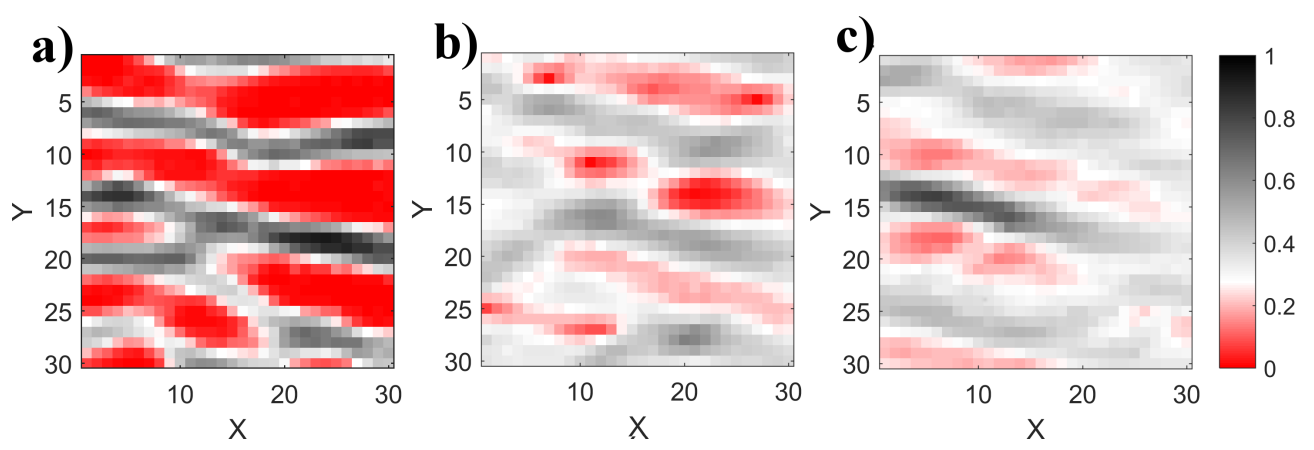

Figure 8: Posterior probability of locating a channel using the SNESIM algorithm with a preferential path, conditional to a) $d 1$, b) $d 2$, and c) $d 3$. 

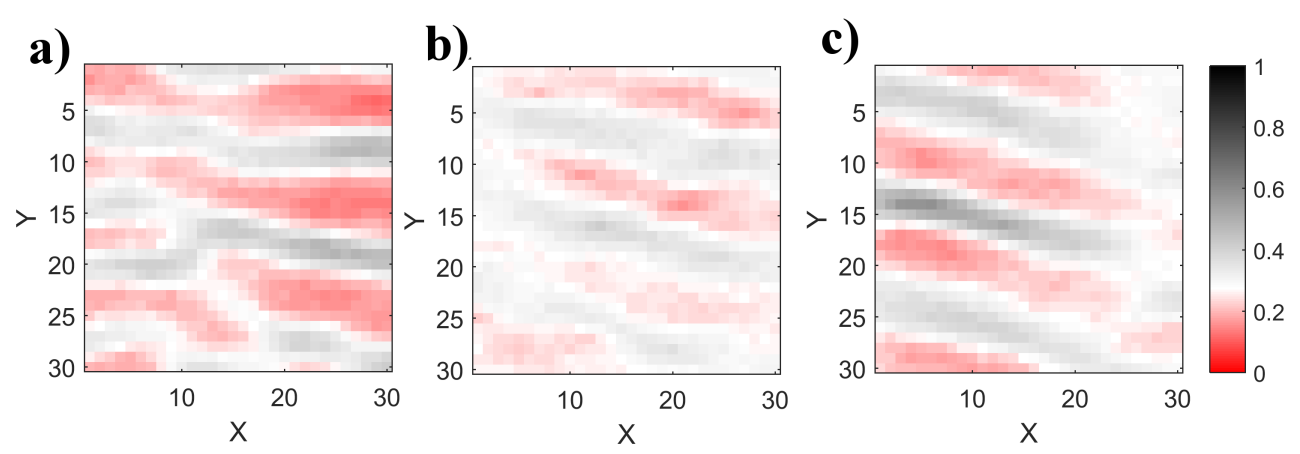

Figure 9: Posterior probability of locating a channel using the DS algorithm using the 3 closest 'soft' data and the 25 closest previously simulated data, with a random path, conditional to a) $d 1$, b) $d 2$, and c) $d 3$. 

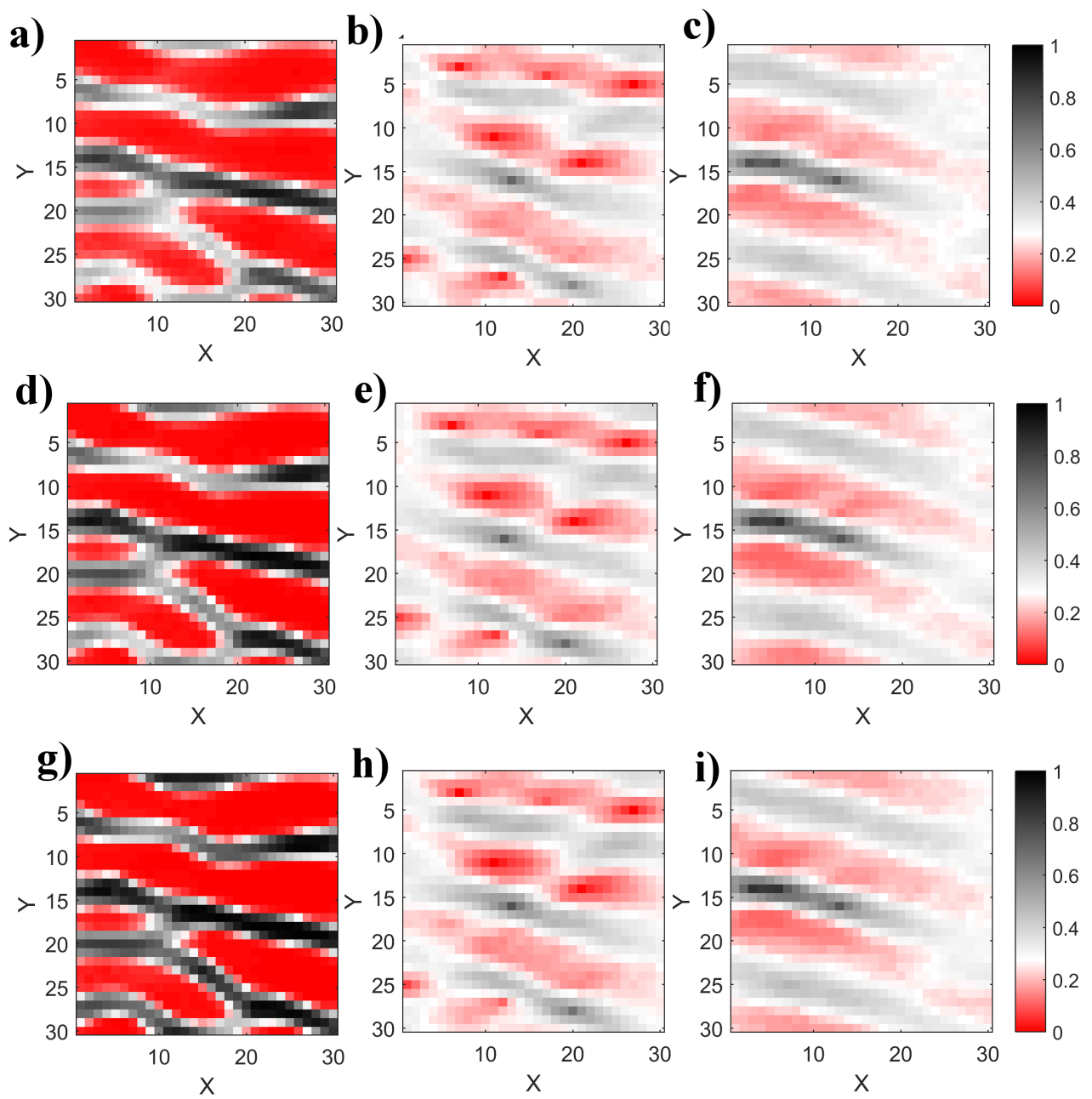

Figure 10: Posterior probability of locating a channel using the DS algorithm using the closest 1 (a,b,c), 3 (d,e,f), and 11 (g,h,i) 'soft'/uncertain data and the 25 closest previously simulated data, with a preferential path, conditional to $d 1$ (a,d,f), $d 2$ (b,e,g), and $d 3$. (c,f,h) 


\section{List of Tables}

1 The relative $\mathrm{L} 2$ norm, $L_{2}\left(P_{m c m c}(\right.$ channel $)-P($ channel $) / L_{2}\left(P_{\text {mcmc }}(\right.$ channel $\left.)\right)$, using the GENESIM algorithm and different choices of simulation paths. The left column indicates the conditional data set considered. Note that the first row for each set of conditional data, refer to unconditional simulation $\left(N_{\text {soft }}=0\right)$, for reference. 'Markov' is marked if the Markov property is assumed such that only co-located data are considered. $N_{\text {soft }}$ indicate the number of closest soft/uncertain data taken into account. The numbers in parentheses is the simulation time in seconds. 36

2 The relative $\mathrm{L} 2$ norm, $L_{2}\left(P_{m c m c}(\right.$ channel $)-P($ channel $) / L_{2}\left(P_{m c m c}(\right.$ channel $\left.)\right)$, using the DS algorithm and different choices of simulation paths. See Table 1 for description. . . . . . . . . . . . 37

3 The relative L2 norm, $L_{2}\left(P_{m c m c}(\right.$ channel $)-P($ channel $) / L_{2}\left(P_{m c m c}(\right.$ channel $\left.)\right)$, using the SNESIM algorithm and different choices of simulation paths. See Table 1 for description. . . . . . . . . . . . . 38 


\begin{tabular}{c|ccccc}
\hline & Markov & $N_{\text {soft }}$ & Unilateral & Random & Preferential \\
\hline$d 1$ & & 0 & $0.78(42.2 \mathrm{~s})$ & $0.77(59.8 \mathrm{~s})$ & $0.77(59.8 \mathrm{~s})$ \\
\hline$d 1$ & $*$ & 1 & $0.63(40.6 \mathrm{~s})$ & $0.70(59.7 \mathrm{~s})$ & $0.43(39.5 \mathrm{~s})$ \\
\hline$d 1$ & & 1 & $0.63(40.7 \mathrm{~s})$ & $0.70(60.0 \mathrm{~s})$ & $0.43(39.7 \mathrm{~s})$ \\
\hline$d 1$ & & 3 & $0.56(44.2 \mathrm{~s})$ & $0.67(60.9 \mathrm{~s})$ & $0.35(40.4 \mathrm{~s})$ \\
\hline$d 1$ & & 11 & $0.47(46.3 \mathrm{~s})$ & $0.65(64.7 \mathrm{~s})$ & $0.25(46.1 \mathrm{~s})$ \\
\hline$d 2$ & & 0 & $0.35(42.3 \mathrm{~s})$ & $0.35(60.4 \mathrm{~s})$ & $0.35(60.6 \mathrm{~s})$ \\
\hline & & & & & \\
\hline$d 2$ & $*$ & 1 & $0.33(42.7 \mathrm{~s})$ & $0.36(60.7 \mathrm{~s})$ & $0.11(59.9 \mathrm{~s})$ \\
\hline$d 2$ & & 1 & $0.24(87.9 \mathrm{~s})$ & $0.27(109.7 \mathrm{~s})$ & $0.11(188.3 \mathrm{~s})$ \\
\hline$d 2$ & & 3 & $0.19(167.0 \mathrm{~s})$ & $0.25(145.7 \mathrm{~s})$ & $0.11(189.7 \mathrm{~s})$ \\
\hline$d 2$ & & 11 & $0.21(284.4 \mathrm{~s})$ & $0.22(189.6 \mathrm{~s})$ & $0.10(191.1 \mathrm{~s})$ \\
\hline$d 3$ & & 0 & $0.35(42.5 \mathrm{~s})$ & $0.35(60.1 \mathrm{~s})$ & $0.35(60.8 \mathrm{~s})$ \\
\hline$d 3$ & $*$ & 1 & $0.34(42.7 \mathrm{~s})$ & $0.36(60.4 \mathrm{~s})$ & $0.10(60.9 \mathrm{~s})$ \\
\hline$d 3$ & & 1 & $0.24(186.3 \mathrm{~s})$ & $0.26(152.0 \mathrm{~s})$ & $0.10(179.7 \mathrm{~s})$ \\
\hline$d 3$ & & 3 & $0.18(273.7 \mathrm{~s})$ & $0.17(180.7 \mathrm{~s})$ & $0.07(178.6 \mathrm{~s})$ \\
\hline$d 3$ & & 11 & $0.18(270.3 \mathrm{~s})$ & $0.18(182.0 \mathrm{~s})$ & $0.07(179.7 \mathrm{~s})$ \\
\hline \hline
\end{tabular}

Table 1: The relative L2 norm, $L_{2}\left(P_{m c m c}(\right.$ channel $)-P($ channel $) / L_{2}\left(P_{m c m c}(\right.$ channel $\left.)\right)$, using the GENESIM algorithm and different choices of simulation paths. The left column indicates the conditional data set considered. Note that the first row for each set of conditional data, refer to unconditional simulation $\left(N_{\text {soft }}=0\right)$, for reference. 'Markov' is marked if the Markov property is assumed such that only co-located data are considered. $N_{\text {soft }}$ indicate the number of closest soft/uncertain data taken into account. The numbers in parentheses is the simulation time in seconds. 


\begin{tabular}{c|ccccc}
\hline & Markov & $N_{\text {soft }}$ & Uni & Random & Preferential \\
\hline$d 1$ & & 0 & $0.78(20.8 \mathrm{~s})$ & $0.77(37.3 \mathrm{~s})$ & $0.77(37.4 \mathrm{~s})$ \\
\hline$d 1$ & $*$ & 1 & $0.61(26.1 \mathrm{~s})$ & $0.67(43.9 \mathrm{~s})$ & $0.42(18.3 \mathrm{~s})$ \\
\hline$d 1$ & & 1 & $0.62(26.2 \mathrm{~s})$ & $0.67(43.7 \mathrm{~s})$ & $0.41(18.5 \mathrm{~s})$ \\
\hline$d 1$ & & 3 & $0.52(51.1 \mathrm{~s})$ & $0.65(74.2 \mathrm{~s})$ & $0.34(19.9 \mathrm{~s})$ \\
\hline$d 1$ & & 11 & $0.56(44.2 \mathrm{~s})$ & $0.67(60.9 \mathrm{~s})$ & $0.35(40.4 \mathrm{~s})$ \\
\hline \hline$d 2$ & & 0 & $0.36(20.7 \mathrm{~s})$ & $0.35(37.3 \mathrm{~s})$ & $0.35(37.4 \mathrm{~s})$ \\
\hline \hline$d 2$ & $*$ & 1 & $0.34(21.7 \mathrm{~s})$ & $0.35(37.3 \mathrm{~s})$ & $0.11(36.7 \mathrm{~s})$ \\
\hline$d 2$ & & 1 & $0.20(30.3 \mathrm{~s})$ & $0.28(53.9 \mathrm{~s})$ & $0.10(45.8 \mathrm{~s})$ \\
\hline$d 2$ & & 3 & $0.16(78.5 \mathrm{~s})$ & $0.22(104.2 \mathrm{~s})$ & $0.11(47.4 \mathrm{~s})$ \\
\hline$d 2$ & & 11 & $0.25(448.8 \mathrm{~s})$ & $0.20(390.4 \mathrm{~s})$ & $0.11(55.7 \mathrm{~s})$ \\
\hline \hline$d 3$ & & 0 & $0.36(19.6 \mathrm{~s})$ & $0.34(38.8 \mathrm{~s})$ & $0.34(37.9 \mathrm{~s})$ \\
\hline \hline$d 3$ & $*$ & 1 & $0.33(19.9 \mathrm{~s})$ & $0.36(39.2 \mathrm{~s})$ & $0.09(38.5 \mathrm{~s})$ \\
\hline$d 3$ & & 1 & $0.24(40.0 \mathrm{~s})$ & $0.25(62.8 \mathrm{~s})$ & $0.08(46.9 \mathrm{~s})$ \\
\hline$d 3$ & & 3 & $0.16(165.9 \mathrm{~s})$ & $0.16(132.8 \mathrm{~s})$ & $0.07(47.7 \mathrm{~s})$ \\
\hline$d 3$ & & 11 & $0.17(163.0 \mathrm{~s})$ & $0.16(135.1 \mathrm{~s})$ & $0.07(47.4 \mathrm{~s})$ \\
\hline \hline
\end{tabular}

Table 2: The relative L2 norm, $L_{2}\left(P_{m c m c}(\right.$ channel $)-P($ channel $) / L_{2}\left(P_{m c m c}(\right.$ channel $\left.)\right)$, using the DS algorithm and different choices of simulation paths. See Table 1 for description. 


\begin{tabular}{c|ccccc}
\hline & Markov & $N_{\text {soft }}$ & Uni & Random & Preferential \\
\hline$d 1$ & & 0 & $0.77(36.0 \mathrm{~s})$ & $0.78(62.6 \mathrm{~s})$ & $0.78(63.2 \mathrm{~s})$ \\
\hline$d 1$ & $*$ & 1 & $0.64(38.2 \mathrm{~s})$ & $0.54(66.1 \mathrm{~s})$ & $0.43(93.1 \mathrm{~s})$ \\
\hline \hline$d 2$ & & 0 & $0.36(35.7 \mathrm{~s})$ & $0.38(63.0 \mathrm{~s})$ & $0.38(62.6 \mathrm{~s})$ \\
\hline$d 2$ & $*$ & 1 & $0.37(36.5 \mathrm{~s})$ & $0.34(64.2 \mathrm{~s})$ & $0.20(69.6 \mathrm{~s})$ \\
\hline \hline$d 3$ & & 0 & $0.34(36.1 \mathrm{~s})$ & $0.36(63.3 \mathrm{~s})$ & $0.36(63.4 \mathrm{~s})$ \\
\hline$d 3$ & $*$ & 1 & $0.36(36.6 \mathrm{~s})$ & $0.35(63.4 \mathrm{~s})$ & $0.16(58.9 \mathrm{~s})$ \\
\hline \hline
\end{tabular}

Table 3: The relative L2 norm, $L_{2}\left(P_{m c m c}(\right.$ channel $)-P($ channel $) / L_{2}\left(P_{m c m c}(\right.$ channel $\left.)\right)$, using the SNESIM algorithm and different choices of simulation paths. See Table 1 for description. 